EPJ manuscript No.

(will be inserted by the editor)

\title{
Quantum kinetic theory of phonon-assisted carrier transitions in nitride-based quantum-dot systems
}

\author{
J. Seebeck ${ }^{1}$, T.R. Nielsen ${ }^{1}$, P. Gartner ${ }^{1,2}$, and F. Jahnke ${ }^{1}$ \\ 1 Institute for Theoretical Physics, University of Bremen, 28334 Bremen, Germany \\ 2 National Institute for Materials Physics, POB MG-7, Bucharest-Magurele, Romania
}

June 23, 2018

\begin{abstract}
A microscopic theory for the interaction of carriers with LO phonons is used to study the ultrafast carrier dynamics in nitride-based semiconductor quantum dots. It is shown that the efficiency of scattering processes is directly linked to quasi-particle renormalizations. The electronic states of the interacting system are strongly modified by the combined influence of quantum confinement and polar coupling. Inherent electrostatic fields, typical for InGaN/GaN quantum dots, do not limit the fast scattering channels.
\end{abstract}

PACS. 7 3.21.La -7 8.67.Hc

\section{Introduction}

In the rapidly evolving field of semiconductor quantum dots (QDs), which provide many new applications in optoelectronics, nitride-based systems are of growing interest due to their large range of possible emission frequencies. [1, , 2,3 The interaction of carriers with LO phonons is an important scattering process which determines the redistribution of carriers between the localized QD states and between QD and wetting-layer (WL) states. Furthermore, these interaction processes lead to optical dephasing which is of central importance in coherent optical experiments and determines the homogeneous broadening of absorption and gain spectra. In nitride-based QDs, the role of interaction with LO phonons is increased due to the intermediate polar coupling strength in this material system. On the other hand, for QDs with wurtzite crystal structure grown along the c-axis, strong built-in fields modify the electronic states via the quantum-confined Stark effect. 4. The aim of this paper is to determine the efficiency of carrier-phonon interaction under these conditions.

While a simplified treatment of carrier-phonon interaction in QDs, based on Fermi's golden rule, leads to the prediction of a phonon-bottleneck (see [5] and references therein), recently it has been emphasized that perturbation theory is not applicable for QD systems and that the interaction needs to be treated in the polaron picture. 6 . 7. A quantum-kinetic theory has been used to describe carrier scattering processes due to QD polarons. 8 , We extend this model by including also the optical generation of carriers by means of a short laser pulse. Naturally linked to this is the dephasing of the coherently driven interband polarization, which is described on the same quantum-kinetic level as the carrier scattering. Further- more, we study intermediate polar coupling and include a self-consistent description of the quantum-confined Stark effect for nitride-based QDs.

\section{Quantum-dot model}

We consider a system of self-assembled InGaN/GaN QDs, grown on the c-plane in wurtzite crystal structure where the spontaneous and piezoelectric polarization along the c-axis cause large electrostatic fields. The calculation of single-particle properties for these QDs is a challenging task on its own. Considerable recent progress has been made in $\boldsymbol{k} \cdot \boldsymbol{p}$ and tight-binding models for realistic QD geometries of various material systems to determine the single-particle wave functions and energies and the resulting free-particle optical transitions. 9, 10,11,12] With the investigation of many-body interaction effects for given single-particle states, this paper follows a different aim. Previous calculations of carrier-carrier 13,14 and carrierphonon 8 scattering in QDs showed that particular details of the wavefunctions are less important, while results are more sensitive to the energy stucture (level spacing and degeneracy).

For our purpose, we use a simplified model for the single-particle states of the QD system based on a separation ansatz $\Phi(\boldsymbol{r})=\varphi(\boldsymbol{\rho}) \xi(z)$ for the wave functions. The in-plane part $\varphi(\boldsymbol{\rho})$ describes either the localized QD states or the delocalized WL states which form a continuum at higher energies. In growth direction, perpendicular to the WL plane, the carrier confinement potential is modified by intrinsic electrostatic fields. Due to the quantum-confined Stark effect, charges become partly separated and a screening field builds up which reduces the 
J. Seebeck et al.: Quantum kinetic theory of phonon-assisted carrier transitions in nitride-based quantum-dot systems

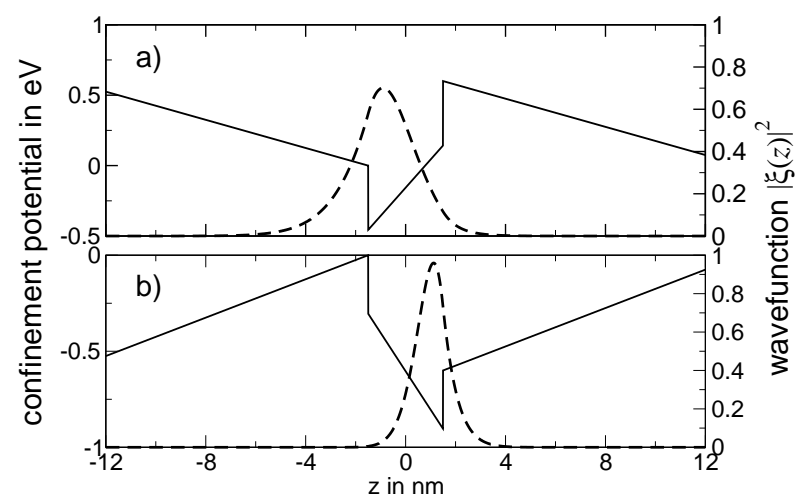

Fig. 1. Confinement potential $U(z)$ (solid line) and wave function $|\xi(z)|^{2}$ (dashed line) for electrons (a) and holes (b) in growth direction for an electrostatic field of $2 \mathrm{MV} / \mathrm{cm}$.

intrinsic electrostatic field. Therefore $\xi(z)$ has to be calculated from a self-consistent solution of the Schrödinger and Poisson equation. For details on the QD model, the calculation of wavefunctions and material parameters, see 14.

We consider QDs with two confined shells which due to their angular momentum properties are referred to as the s-shell (ground state) and the p-shell (excited state). The level spacing between s- and p-states is $1.2 \hbar \omega_{\mathrm{LO}}$ for electrons and $0.4 \hbar \omega_{\mathrm{LO}}$ for holes. For the intrinsic electrostatic field, we consider a typical field strength of $2 \mathrm{MV} / \mathrm{cm}$.

\section{Many-body theory}

A perturbative treatment of the carrier interaction with LO phonons is only possible when modifications of the single-particle states and energies due to the polar coupling remain small. In QDs the localized states with discrete energies are subject to hybridization effects even in materials with weak polar coupling. 6, 7, 8. The QD polaron states are broadened due to the interaction with carriers in the WL continuum, which ensures dissipation. 8 Quasi-particle renormalizations can be described using the retarded Green's function, which obeys the Dyson equation

$$
\begin{aligned}
& {\left[i \hbar \frac{\partial}{\partial \tau}-\epsilon_{\alpha}^{\mathrm{c}}\right] G_{\alpha}^{\mathrm{c}, \mathrm{R}}(\tau)=\delta(\tau)} \\
& +\int_{0}^{\tau} d \tau^{\prime} \sum_{\beta} G_{\beta}^{\mathrm{c}, \mathrm{R}}\left(\tau-\tau^{\prime}\right) D_{\alpha \beta}^{\mathrm{cc},>}\left(\tau-\tau^{\prime}\right) G_{\alpha}^{\mathrm{c}, \mathrm{R}}\left(\tau^{\prime}\right)
\end{aligned}
$$

for electrons in the conduction band and a similar equation with $D^{\mathrm{vv},<}$ for the valence band. This equation describes the polaron in the self-consistent, diagonal, random-phase approximation (RPA) 15 8. The single-particle energies for either localized or extended states are given by $\epsilon_{\alpha}^{\lambda}$, with $\lambda=\mathrm{c}, \mathrm{v}$ being the band index. Here, the corresponding wave functions (which incorporate effects of the builtin field) enter via the interaction matrix elements $M_{\alpha, \beta}^{\lambda}$.
They are included in the phonon propagator

$$
\begin{aligned}
D_{\alpha \beta}^{\lambda \mu, \gtrless}(\tau) & =\sum_{\boldsymbol{q}} M_{\alpha \beta}^{\lambda}(\boldsymbol{q}) M_{\beta \alpha}^{\mu}(-\boldsymbol{q}) \\
& \times\left[N_{\mathrm{LO}} e^{ \pm i \omega_{\mathrm{LO}} \tau}+\left(1+N_{\mathrm{LO}}\right) e^{\mp i \omega_{\mathrm{LO}} \tau}\right]
\end{aligned}
$$

where $N_{\mathrm{LO}}$ is a Bose-Einstein function for the population of the phonon modes (assumed to act as a bath in thermal equilibrium) and $\omega_{\mathrm{LO}}$ is the LO-phonon frequency. The Fourier transform of the retarded GF directly provides the spectral function $\widehat{G}_{\alpha}(\omega)=-2 \operatorname{Im} G_{\alpha}^{\mathrm{R}}(\omega)$ which reflects the density of states (DOS) for a carrier in state $\alpha$.

For non-interacting carriers, the spectral function contains $\delta$-functions at the free-particle energies, reflecting the infinite lifetime of these states (if recombination on a much longer timescale is neglected). The spectral functions for carriers in the QD-WL system interacting with lattice distortions are shown in Fig. 2 For a WL electron with zero momentum (at the band edge), the $\delta$-like spectral function in the absence of interaction (indicated by a vertical line in Fig. 2a) is broadened. The corresponding finite lifetime of the state is due to possible scattering events which are self-consistently calculated in terms of renormalized states and in arbitrary order in the interaction by means of a direct numerical solution of Eqs. (1) and (2). Furthermore, the energy lowering of the interacting state (polaron shift) and weak LO phonon satellites are obtained.

For the discrete QD states, Figs. 2b) and c) show that the free-particle picture is strongly modified. On this level, a direct comparison between the the weak-polar coupling material system InGaAs (Fig. 1 of Ref. 8]) and the InGaN system with intermediate polar coupling is possible. In the first case, one can clearly distinguish between phonon replicas of the QD states which are spaced by the LO-phonon energy $\hbar \omega_{\text {LO }}$ around the central peak, and the hybridization effect which leads to a splitting of these peaks that is smaller than the LO-phonon energy (when the detuning between the QD level spacing and the LOphonon energy is small). In the InGaN material system, the hybridization is much stronger and one can no longer distinguish between phonon replicas and their splitting. Moreover, the obtained rich multi-peak structure provides a stronger overlap between the spectral functions which increases the scattering efficiency discussed below. Broadening of the resonances is provided by the coupling to the WL states and the finite LO-phonon lifetime (assumed to be $5 \mathrm{ps})$.

In the markovian limit, the transition rate between two states $\alpha$ and $\beta$ is proportional to the overlap $\Lambda_{\alpha \beta}=$ $\int d \omega \widehat{G}_{\alpha}(\omega) \widehat{G}_{\beta}\left(\omega \pm \omega_{\mathrm{LO}}\right)$. In the free-particle picture, considering the $\delta$-like DOS, the results of Fermi's golden rule are recovered. The level-spacing has to match exactly the LO-phonon energy $\hbar \omega_{\mathrm{LO}}$, otherwise the overlap vanishes and carrier transitions are not possible. In the polaron picture, strong overlap between the spectral functions of the QD states as well as between the QD and WL states is obtained which already indicates the possibility of efficient scattering processes. On ultrafast timescales, the 


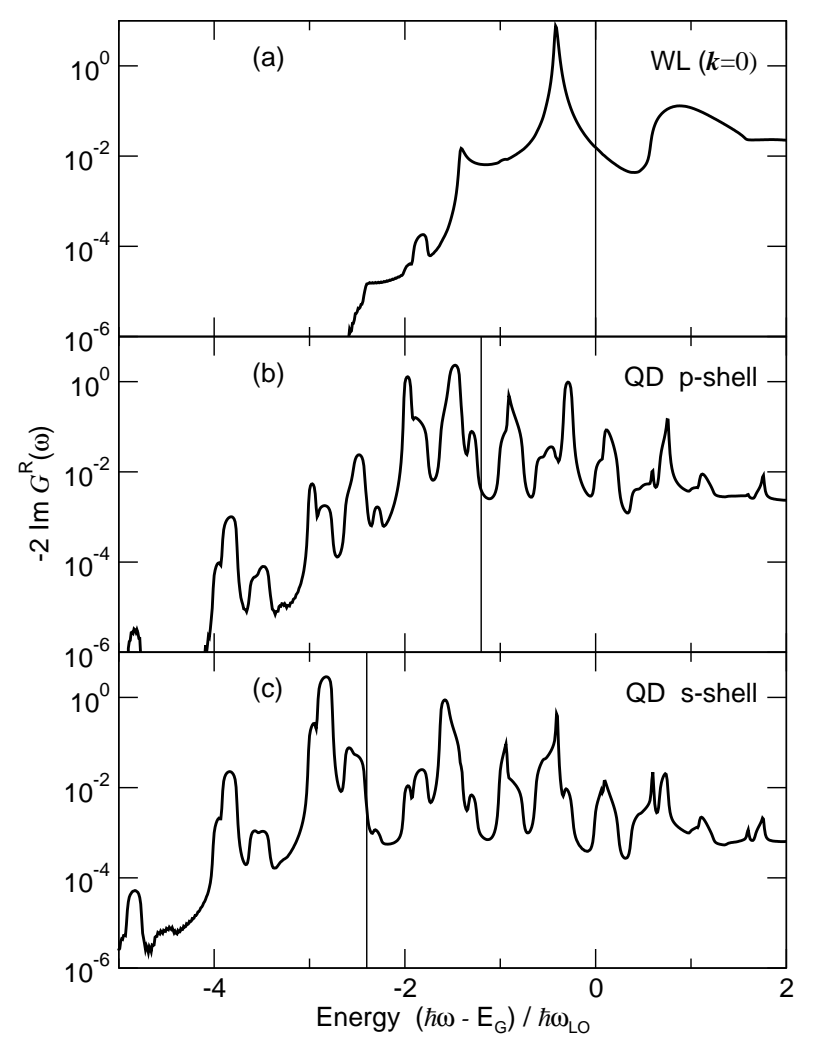

Fig. 2. Spectral function for a WL electron with zero momentum (a) and for a QD electron in the p-shell (b) and in the sshell (c). Vertical lines indicate the positions of the $\delta$-functions for a non-interacting carriers.

Markov approximation is not valid and quantum-kinetic effects additionally increase the scattering rates.

To study the carrier relaxation from p-shell into s-shell, we consider the optical excitation of carriers with a short (100 fs) laser pulse having spectral overlap only with the pshell. The coupled carrier and polarization dynamics are described by the kinetic equation for the single-particle density matrix $\rho_{\alpha}(t)$, which contains optically induced interband transition amplitudes $\psi_{\alpha}$ and occupation probabilities $f_{\alpha}^{c, v}$ according to [16]

$$
\begin{aligned}
\frac{\partial}{\partial t} \rho_{\alpha}(t) & =\frac{\partial}{\partial t}\left(\begin{array}{cc}
f_{\alpha}^{\mathrm{c}} & \psi_{\alpha} \\
\psi_{\alpha}^{*} & f_{\alpha}^{\mathrm{v}}
\end{array}\right)= \\
& =\left[\left(\begin{array}{cc}
\tilde{\epsilon}_{\alpha}^{\mathrm{c}} & -\Omega_{\alpha} \\
-\Omega_{\alpha}^{*} & \tilde{\epsilon}_{\alpha}^{\mathrm{v}}
\end{array}\right), \rho_{\alpha}\right]+\left.\frac{\partial}{\partial t} \rho_{\alpha}(t)\right|_{\text {coll. }} .
\end{aligned}
$$

In this matrix notation of the semiconductor Bloch equations, Hartree-Fock renormalizations due to the carrier Coulomb interaction are included in the single-particle energies $\tilde{\epsilon}_{\alpha}^{\lambda}=\epsilon_{\alpha}^{\lambda}+\sum_{\beta} v_{\alpha \beta}^{\lambda \lambda}\left(f_{\alpha}^{\lambda}-\delta_{\lambda \mathrm{v}}\right)$ and in the Rabi energy $\Omega_{\alpha}=\boldsymbol{d} \boldsymbol{E}(t)+\sum_{\beta} v_{\alpha \beta}^{\mathrm{cv}} \psi_{\alpha}$. Here $\boldsymbol{d}$ is the dipole coupling, $\boldsymbol{E}(t)$ the laser pulse and $v_{\alpha \beta}^{\lambda \lambda^{\prime}}$ are the matrix elements of the Coulomb interaction [14. The quantum kinetic description of the carrier-phonon interaction, based on the generalized Kadanoff-Baym ansatz [16],

$$
\begin{aligned}
&\left.\frac{\partial}{\partial t} \rho_{\alpha}^{\lambda \lambda^{\prime}}(t)\right|_{\text {coll. }}=\int_{-\infty}^{t} d t^{\prime} \sum_{\beta \mu} \\
& \times\left\{G_{\beta}^{\lambda, \mathrm{R}}\left(t, t^{\prime}\right)\left[G_{\alpha}^{\lambda^{\prime}, \mathrm{R}}\left(t, t^{\prime}\right)\right]^{*}\right. \\
& \times {\left[-\left[\delta_{\lambda \mu}-\rho_{\beta}^{\lambda \mu}\left(t^{\prime}\right)\right] \rho_{\alpha}^{\mu \lambda^{\prime}}\left(t^{\prime}\right) D_{\alpha \beta}^{\lambda \mu,>}\left(t, t^{\prime}\right)\right.} \\
&\left.+\rho_{\beta}^{\lambda \mu}\left(t^{\prime}\right)\left[\delta_{\mu \lambda^{\prime}}-\rho_{\alpha}^{\mu \lambda^{\prime}}\left(t^{\prime}\right)\right] D_{\alpha \beta}^{\lambda \mu,<}\left(t, t^{\prime}\right)\right] \\
&+ G_{\alpha}^{\lambda, \mathrm{R}}\left(t, t^{\prime}\right)\left[G_{\beta}^{\lambda^{\prime}, \mathrm{R}}\left(t, t^{\prime}\right)\right]^{*} \\
& \times+\left[\delta_{\lambda \mu}-\rho_{\alpha}^{\lambda \mu}\left(t^{\prime}\right)\right] \rho_{\beta}^{\mu \lambda^{\prime}}\left(t^{\prime}\right) D_{\alpha \beta}^{\mu \lambda^{\prime},>}\left(t, t^{\prime}\right) \\
&\left.\left.-\rho_{\alpha}^{\lambda \mu}\left(t^{\prime}\right)\left[\delta_{\mu \lambda^{\prime}}-\rho_{\beta}^{\mu \lambda^{\prime}}\left(t^{\prime}\right)\right] D_{\alpha \beta}^{\mu \lambda^{\prime},<}\left(t, t^{\prime}\right)\right]\right\},
\end{aligned}
$$

represents a generalization of the formulation in Ref. 8 to the coupled carrier and polarization dynamics and describes scattering, dephasing, and energy renormalization contributions. The RPA self-energy was used, and Hartree terms were neglected, since we consider only low-density populations. In the markovian limit, the slowly varying part of $\rho\left(t^{\prime}\right)$ is taken out of the integral and memory kernels of the type $\Lambda_{\alpha \beta}^{\lambda \mu}=\int d t^{\prime} G_{\alpha}^{\lambda, \mathrm{R}}\left(t^{\prime}\right)\left[G_{\beta}^{\mu, \mathrm{R}}\left(t^{\prime}\right)\right]^{*} e^{ \pm i \omega_{\mathrm{LO}} t^{\prime}}$ determine the scattering efficiency between two states $\alpha$ and $\beta$ according to the overlap of spectral functions in the frequency domain, as stated above. In the non-markovian case, the memory kernel contains also the single particle density matrix. This gives rise to memory effects since the actual time evolution depends explicitly on the history of the system.

The considered level-spacing of $1.2 \hbar \omega_{\mathrm{LO}}$ for electrons and $0.4 \hbar \omega_{\mathrm{LO}}$ for holes provides no carrier transitions in a free-particle picture. Results of the quantum-kinetic calculation are shown in Fig. [3] Due to pulse excitation, first the p-shell is populated. The carrier relaxation is so fast that already during the optical pulse a strong redistribution from the p-shell to the s-shell takes place. The ultrafast scattering processes are connected with strong dephasing of the coherent interband polarization, which is included on the same quantum-kinetic level in Eq. (4). The strong dephasing leads to a time evolution of the interband polarization, which practically follows the optical pulse (not shown). Correspondingly, the coherent regime is limited to the duration of the $100 \mathrm{fs}$ pump pulse. A transient strong increase of the total carrier density is obtained during the coherent regime, as can be seen in Fig. 3 b).

The time-domain oscillations in Fig. 3a) indicate the strong coupling between carriers and phonons. The oscillation frequency is connected to the hybridization in the DOS. The phenomenon is analogous to Rabi-oscillations, though not due to coupling of carriers to the optical field, as recently discussed for QDs in Refs. 17 18, but rather due to coupling of carriers to the bosonic field of the phonons. Note that the oscillations appear after the optical pump pulse in the incoherent regime. Hence they are not connected to optical coherence. At larger times, 

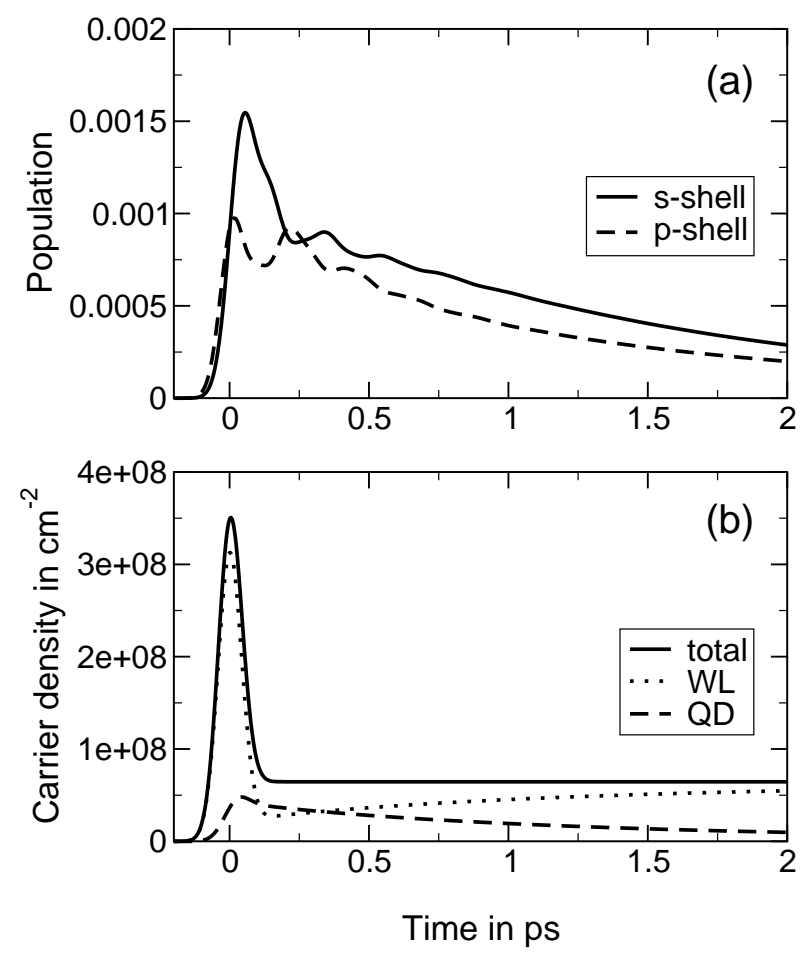

Fig. 3. (a) Time evolution of the electron population in the p-shell (dashed line) and s-shell (solid line) after optical excitation with a 100 fs pulse resonant with the p-shell.(b) Total carrier density (solid line), and carrier density in the WL (dotted line) and QD (dashed line) versus time.

the possibility of carrier transitions between QD and WL states leads to a redistribution of carriers from the QD states into the WL. The corresponding reduction of the QD population in connection with the increasing WL carrier density can be seen in Figs. 3 a) and b), respectively. Of course, the total carrier density remains constant after the pulse.

The ultrafast scattering and dephasing processes are the direct result of the polaronic renormalizations. Only within a non-markovian calculation the above mentioned oscillations in connection with the strong-coupling regime for the carrier-phonon interaction can be obtained. [8] In this respect, there is no qualitative difference between the InGaAs and InGaN material systems. In the latter case, the larger polar coupling increases the scattering efficiency while the electron and hole charge separation induced by the built-in fields reduces only inter-band but not intraband processes.

In the presented example, a weak excitation pulse has been used to justify the restriction of the interaction to LO phonons. At elevated excitation densities, carrier-carrier scattering additionally contributes to fast scattering processes. 14. carriers with LO phonons. Therefore, Fermi's golden rule is not applicable and a quantum-kinetic description is necessary, which provides ultrafast scattering channels.

This work was supported by the Deutsche Forschungsgemeinschaft. We acknowledge a grant for CPU time at the NIC, Forschungszentrum Jülich.

\section{References}

1. S. Nakamura, G. Fasol, and S. J. Pearton. The blue laser diode. Springer, Berlin, 2000.

2. Y.-H. Cho, B. J. Kwon, J. Barjon, J. Brault, B. Daudin, H. Mariette, and L. S. Dang. Appl. Phys. Lett., 81:4934, 2002.

3. S. Kalliakos, T. Bretagnon, P. Lefebvre, T. Taliercio, B. Gil, N. Grandjean, B. Damilano, A. Dussaigne, and J. Massies. J. Appl. Phys., 96:180, 2004.

4. F. Bernardini and V. Fiorentini. Phys. Rev. B, 57:R9427, 1998.

5. H. Benisty. Phys. Rev. B, 51:13281, 1995.

6. T. Inoshita and H. Sakaki. Phys. Rev. B, 56:R4355, 1997.

7. O. Verzelen, R. Ferreira, G. Bastard, T. Inoshita, and H. Sakaki. phys. stat. sol. (a), 190:213, 2002.

8. J. Seebeck, T.R. Nielsen, P. Gartner, and F. Jahnke. Phys. Rev. B, 71:125327, 2005.

9. V. Ranjan, G. Allan, C. Priester, and C. Delerue. Phys. Rev. B, 68:115305, 2003.

10. A. Bagga, P.K. Chattopadhyay, and S. Ghosh. Phys. Rev.

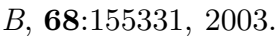

11. V.A. Fonoberov and A.A. Balandin. J. Appl. Phys., 94:7178, 2003.

12. A.D. Andreev and E.P. O'Reilly. Phys. Rev. B, 62:15851, 2000 .

13. T.R. Nielsen, P. Gartner, and F. Jahnke. Phys. Rev. B, 69:235314, 2004.

14. T.R. Nielsen, P. Gartner, M. Lorke, J. Seebeck, and F. Jahnke. Phys. Rev. B, 72:235311, 2005.

15. K. Král and Z. Khás. Phys. Rev. B, 57:R2061, 1998.

16. H. Haug and A.-P. Jauho. Quantum Kinetics in Transport E Optics of Semiconductors. Springer-Verlag, Berlin, 1. edition, 1996.

17. J. Förstner, C. Weber, J. Danckwerts, and A. Knorr. Phys. Rev. Lett., 91:127401, 2003.

18. Q.Q. Wang, A. Muller, P. Bianucci, E. Rossi, Q.K. Xue, T. Takagahara, C. Piermarocchi, A.H. MacDonald, and C.K. Shih. Phys. Rev. B, 72:35306, 2005.

\section{Conclusion}

It has been shown that in nitride-based QD systems with intermediate polar coupling strong modifications of the free-particle states are obtained due to the interaction of 